Cahiers $d u$ MONDE RUSSE

\section{Cahiers du monde russe}

Russie - Empire russe - Union soviétique et États indépendants

48/2-3 | 2007

Les résonances de 1905

\title{
Une révolution sans révolutionnaires1905 dans des manuels russes récents
}

Jutta SCHERRER

\section{OpenEdition}

\section{Journals}

Édition électronique

URL : https://journals.openedition.org/monderusse/9025

DOI : 10.4000/monderusse.9025

ISSN : $1777-5388$

Éditeur

Éditions de l'EHESS

\section{Édition imprimée}

Date de publication : 15 avril 2007

Pagination : $539-556$

ISBN : 978-2-7132-2147-7

ISSN : $1252-6576$

Référence électronique

Jutta SCHERRER, "Une révolution sans révolutionnaires 1905 dans des manuels russes récents », Cahiers du monde russe [En ligne], 48/2-3 | 2007, mis en ligne le 01 janvier 2007, consulté le 04 septembre 2022. URL : http://journals.openedition.org/monderusse/9025 ; DOI : https://doi.org/ 10.4000/monderusse. 9025 
chercher : repérer : avancer

Cet article est disponible en ligne à l'adresse :

http://www.cairn.info/article.php?ID REVUE=CMR\&ID NUMPUBLIE=CMR 482\&ID ARTICLE=CMR 4820539

\title{
Une révolution sans révolutionnaires1905 dans des manuels russes récents
}

\author{
par Jut ta SCHERRER
}

\section{Editions de l'EHESS | Cahiers du monde russe}

\author{
$2007 / 2-3-$ Vol 48 \\ ISSN 1252-6576 | ISBN 9782713221477 | pages 539 à 556
}

Pour citer cet article :

—SCHERRER J., Une révolution sans révolutionnaires1905 dans des manuels russes récents, Cahiers du monde russe 2007/ 2-3, Vol 48, p. 539-556.

Distribution électronique Cairn pour les Editions de l'EHESS.

(C) Editions de l'EHESS. Tous droits réservés pour tous pays.

La reproduction ou représentation de cet article, notamment par photocopie, n'est autorisée que dans les limites des conditions générales d'utilisation du site ou, le cas échéant, des conditions générales de la licence souscrite par votre établissement. Toute autre reproduction ou représentation, en tout ou partie, sous quelque forme et de quelque manière que ce soit, est interdite sauf accord préalable et écrit de l'éditeur, en dehors des cas prévus par la législation en vigueur en France. Il est précisé que son stockage dans une base de données est également interdit. 


\section{UNE RÉVOLUTION SANS RÉVOLUTIONNAIRES 1905 DANS DES MANUELS RUSSES RÉCENTS}

La Russie ne présente pas un cas à part : les manuels d'histoire marquent partout un retard considérable par rapport à la recherche historique de pointe. Ceci concerne notamment le traitement de l'histoire du XX $x^{\mathrm{e}}$ siècle. Dans des situations de ruptures socio-politiques et culturelles, les manuels d'histoire sont des indicateurs particulièrement révélateurs du changement des paradigmes. Rappelons l'année 1988 en URSS, quand tous les examens d'histoire du $\mathrm{XX}^{\mathrm{e}}$ siècle furent suspendus dans l'enseignement secondaire et à l'entrée des facultés d'histoire. Un tel phénomène s'était déjà produit après les révélations de Hruščev au XXe congrès du PCUS. Mais, sans doute, pourrions-nous aussi renvoyer à des exemples occidentaux : combien de temps fallut-il pour que les camps de concentration du régime nazi fissent partie de l'enseignement de l'histoire dans les écoles secondaires en Allemagne ou la guerre d'Algérie en France !

Dans cette étude, nous nous proposons d'analyser les manuels d'histoire russe de cette dernière décennie et de nous interroger sur la façon dont la première révolution russe de 1905-1907 est traitée et évaluée dans des manuels destinés à l'enseignement de l'histoire dans les dernières classes du lycée et la première année de l'université. Nous prenons pour exemples des manuels trouvés au hasard des librairies russes. Celles-ci disposent actuellement d'un nombre considérable de manuels, au point que le président Vladimir Putin tenta à plusieurs reprises d'user de son influence pour renforcer leur contenu patriotique et proposa même l'introduction d'un manuel d'histoire uniformisé pour tout le pays - tentative jusqu'à présent déjouée par nos collègues russes. Nous avons également pris en considération des manuels conçus pour les enseignants (rédigés par les mêmes auteurs que ceux des

Pour les références complètes des manuels et encyclopédie cités, se reporter à la bibliographie située en fin d'article. 
manuels destinés aux élèves) qui comportent des recommandations méthodologiques et des suggestions sur la façon d'expliquer certains thèmes aux élèves, ainsi que des recueils et anthologies de documents historiques, qui accompagnent certains manuels. De même, nous avons examiné des brochures ou fascicules composés de questions et de réponses pour la préparation aux examens (ekzamennye voprosy / bilety $i$ otvety) rédigés par quelques auteurs en complément de leurs manuels. Des manuels d'histoire de civilisation russe, reconnue depuis quelques années comme sous-discipline de l'histoire par les standarty (programmes obligatoires) du ministère de l'Éducation de la Fédération de Russie, présentent également le plus grand intérêt pour notre propos. Enfin, nous avons examiné une encyclopédie historique conçue pour les élèves (Bólšaja škol'naja enciklopedija «Russika») dont un volume entier est consacré à l'histoire russe au $\mathrm{XX}^{\mathrm{e}}$ siècle ${ }^{1}$. Tous les manuels et documentations utilisés pour notre enquête font l'objet d'une liste chronologique située à la fin de cet article. La plupart sont d'ailleurs recommandés pour l'enseignement par le ministère de l'Éducation de la Fédération de Russie.

À titre de curiosité, notons qu'aucun des collègues historiens russes n'a voulu ni pu nous recommander un manuel d'histoire dont l'approche et le contenu auraient été satisfaisants, à ses yeux, c'est-à-dire qui auraient correspondu aux critères de la recherche historique la plus récente. Aussi critiques soient-ils à l'égard des manuels existants, presque aucun des historiens reconnus n'aimerait prendre le risque d'en rédiger un lui-même. Ce qui nous conduit immédiatement à nous demander qui sont les auteurs des manuels ? Pour la plupart, les manuels sont rédigés par des collectifs d'historiens peu connus par leur recherche. Mais nous dénombrons aussi des directeurs des instituts les plus prestigieux de l'Académie des sciences de Russie qui exercent la responsabilité d'éditeurs de manuels comme Aleksandr Čubarjan (Institut d'histoire universelle) ou Andrej Saharov (Institut d'histoire russe). Étant donné le tirage important des manuels, - ils se vendent par dizaines de milliers d'exemplaires -, l'aspect financier n'est sans doute pas négligeable non plus pour leurs auteurs.

Nous ne nous attarderons pas ici sur les origines académiques ou encore les orientations politiques des auteurs des manuels car notre intention consiste en premier lieu à identifier les tendances dominantes et leur explication de la révolution de 19051907. Il ne s'agit pas non plus de critiquer les personnes responsables des manuels : nous n'ignorons pas les difficultés énormes auxquelles se heurtèrent les historiens russes après la dissolution de l'URSS et l'abandon de l'idéologie régnante. Il n'était guère possible d'acquérir en peu d'années de nouvelles approches et méthodes ni de se tenir au courant des recherches les plus récentes. Nous pouvons nous imaginer les conditions difficiles dans lesquelles furent conçus les nouveaux manuels. Par contre, nous ignorons le rôle de l'autocensure que s'imposèrent les auteurs, en particulier

1. A.V. Dienko, «Revoljucija 1905-1907 gg., 1-ja russkaja revoljucija » [La révolution de 1905-1907, première révolution russe] in Bol'šaja škol'naja enciklopedija «Russika, 2002, p. $387-390$. 
pendant les premières années qui suivirent le Grand Tournant et qu'ils s'imposent peut-être de nouveau aujourd'hui. Nous ne disposons pas non plus des informations sur les lois du marché des livres qui définissent non seulement leur distribution, mais peut-être aussi leur orientation historique et politique. Notons seulement que jusqu'à présent, chaque établissement scolaire est libre en principe de choisir le type de manuel souhaité par ses enseignants, pourvu que l'établissement puisse se permettre l'achat des manuels les plus récents et toujours plus chers.

Dans ce court survol, qui est loin d'être systématique, il s'agit avant tout d'examiner comment sont traitées (ou ignorées) dans les manuels quelques questions clefs concernant la révolution de 1905-1907 et dans quelle mesure la présentation et l'interprétation de cet événement sont supposées contribuer à l'éducation civique du jeune lycéen ou étudiant russe, comme par exemple :

1. la définition de la révolution 1905, sa mise en perspective avec la révolution de 1917 et sa comparaison avec des révolutions européennes précédentes ;

2. les conflits sociaux qui ont conduit aux événements de 1905 et les classes et groupes sociaux (ouvriers, paysans, intelligentsia) en tant qu'acteurs de la révolution ;

3. les acquis de la révolution de 1905, comme le parlementarisme et le multipartisme ;

4. les processus révolutionnaires dans les périphéries de l'Empire comme en Pologne, Finlande, aux pays Baltes et au Caucase ;

5. la narration, la documentation et l'iconographie des manuels ;

6. en quoi les manuels actuels se différencient des manuels soviétiques.

Le fait que la révolution de 1905 ne soit pas thématisée dans les standarty pour l'enseignement de l'histoire russe (otečestvennaja istorija) ${ }^{2}$ est révélateur. Bien que la modernisation industrielle (industrial'naja modernizacija) et les partis politiques de la Russie (političeskie partii Rossii) figurent dans la rubrique des gosstandarty «La Russie du début du XX $X^{\mathrm{e}}$ siècle » (Rossija v načale XXV.), la révolution de 1905 elle-même n'y est pas mentionnée. De « révolution » il n'est question que dans la rubrique suivante qui, cependant, se réfère directement à l'année 1917. C'est ici peut-être la plus grande «déviation » par rapport à l'historiographie soviétique pour laquelle, en suivant l'interprétation obligatoire donnée par Lenin, la révolution de 1905 était la « répétition générale » (general'naja repeticija ) de celle d'octobre 1917.

Pour l'observatrice externe que nous sommes, il est incontestablement difficile sinon impossible d'apprécier le rôle véritable que jouent les gosstandarty dans la conception des manuels ainsi que dans l'enseignement concret de l'otečestvennaja istorija. Il nous est également difficile de savoir dans quelle mesure les maisons d'édition exercent, sous la pression du marché peut-être, une certaine influence sur

2. Ministerstvo obrazovanija Rossijskoj Federacii, Gosudarstvennyj obrazovatel'nyj standart vysšego professional'nogo obrazovanija : Special'nost' 020700 - «Istorija » [Programme obligatoire d'État de l'éducation professionnelle supérieure. Spécialité 020700, Histoire] (section Otečestvennaja istorija GSE.F.03). 
les choix des auteurs des manuels et leurs orientations politiques : il est évident que des manuels reflètent un certain spectre allant des positions libérales jusqu'aux orientations conservatrices et nationales.

En supposant, peut-être un peu naïvement, qu'une encyclopédie historique, conçue spécialement pour les besoins de l'enseignement secondaire, présenterait de la manière la plus évidente les connaissances historiques qu'on souhaite transmettre aux élèves, regardons tout d'abord comment la révolution de 1905 est traitée dans la Bol'šaja škol'naja enciklopedija «Russika», dont un volume entier est consacré à l'histoire de la Russie au XX $\mathrm{XX}^{\mathrm{e}}$ siècle ${ }^{3}$. Dès la première phrase de l'entrée «La révolution de 1905-1907 » («Revoljucija 1905-1907 gg. ») la «Première Révolution russe » est appellée « révolution démocratique en Russie », bien que dans la suite on parle, comme auparavant dans l'historiographie soviétique, d'une « révolution démocratique-bourgeoise ». Les nuances entre ces adjectifs ne sont pas expliquées, pas plus que le terme de révolution lui-même n'est commenté. La révolution est simplement caractérisée comme un résultat de l'aggravation aiguë des contradictions entre l'autocratie et «pratiquement toutes les couches de la société », c'est-à-dire entre «propriétaires terriens » (pomeščiki) et paysans, bourgeoisie et prolétariat, administration tsariste et les populations des «périphéries nationales » (nacional'nye okrainy). De même, la « grande bourgeoisie commerciale et industrielle libérale » qui, selon cette Encyclopédie, se composait avant tout de l'intelligentsia, se trouvait dans une opposition modérée (umerennaja opposicija) par rapport aux structures existantes. La Guerre russo-japonaise n'avait que renforcé la détérioration de la situation politique dans le pays et ainsi rendu évidente la nécessité des réformes politiques et économiques. La classe ouvrière est citée au premier rang des participants actifs de la révolution, suivie par la paysannerie, l'armée et la marine de guerre. Leurs dirigeants ou les mouvements politiques qui les soutenaient restent cependant tout à fait dans l'ombre.

La périodisation de la révolution est divisée en deux étapes : son « ascension » ou pod"em (jusqu'en décembre 1905) et son « déclin » ou spad (jusqu'à la fin juin 1907). Dans la première étape, il est fait état des mouvements de grèves qui donnèrent naissance à des unions professionnelles et politico-professionnelles, aux premiers soviets des députés ouvriers ainsi qu'aux partis libéraux et conservateurs. La seconde étape, malgré le « déclin » des mouvements socio-politiques, se caractérise par le «niveau élevé » des grèves, des mouvements paysans et du mouvement national de libération ainsi que de l'agitation dans l'armée et la marine. La consolidation du champ contre-révolutionnaire, la répression sanglante des participants des révoltes, la dissolution de la Deuxième Douma conduisent à l'étouffement de la révolution et l'autocratie est forcée de créer un parlement, d'admettre des partis et d'autres structures de la société civile (graždanskoe obščestvo), qui accélérèrent l'évolution progressive du capitalisme en Russie.

3. « Revoljucija 1905-1907 gg., 1-ja russkaja revoljucija » in Bol`šaja škol’naja enciklopedija «Russika ».p. 387-390. 
La révolution de 1905 n'est expliquée ici ni sur l'arrière-fond du développement historique de la Russie ni du point de vue de son évolution politique et sociale ultérieure. Plus de la moitié de cette courte entrée - une page entière - est consacrée à une représentation graphique (datant manifestement de l'époque soviétique) de la répartition du mouvement révolutionnaire dans la Russie d'Europe, ainsi qu'à cinq tableaux artistiques aux sujets révolutionnaires conçus pendant les années 1905 1907 et les années vingt. Des renseignements sur le « Dimanche rouge » ou sur la convocation de la Première Douma doivent être cherchés dans des entrées spécifiques, auxquelles celle sur 1905 ne fait pourtant aucun renvoi. Ceci concerne également l'activité des partis et des syndicats ainsi que leurs dirigeants. Le premier soviet des députés ouvriers n'apparaît nulle part dans l'encyclopédie comme entrée indépendante; ainsi l'élève n'apprend même pas l'origine du terme «Union Soviétique ». Le rôle de Lenin dans la révolution (il rentra illégalement en Russie en novembre 1905 et quitta le pays de nouveau en décembre 1907) ne se trouve évoqué que dans l'entrée spécifique « Lenin V.I. ». Ici l'élève peut lire que Lenin a « orienté les bolcheviks vers l'insurrection armée » et «la création d'une république démocratique $»^{4}$.

Faisons un saut et passons de cette présentation, plutôt sèche et guère capable de transmettre à l'élève le véritable impact de la révolution sur la société russe et l'évolution socio-politique des années suivantes, au manuel édité par A.O. Čubarjan, destiné à l'onzième classe du lycée : Otečestvennaja istorija $X X$ - načala $X X I$ veka. Le chapitre « Pervyj revoljucionnyj razlom. 1905-1907gg. » ${ }^{5}$ commence par présenter dans des sous-articles courts et succincts une description des actions des ouvriers, des paysans, de l'armée et de la marine ainsi que des agitations dans les «périphéries nationales ». Il est rappelé que des grèves existaient déjà avant 1905, mais qu'elles étaient principalement limitées aux revendications économiques, telle la manifestation des ouvriers du 9 janvier 1905 sous la conduite de Gapon, dont les participants reconnaissaient le pouvoir du monarque, mais demandaient aussi, outre des améliorations économiques, la convocation des représentants du peuple pour la conduite des affaires de l'État. « Mais le pouvoir suprême n'était pas prêt pour un dialogue avec le peuple. »

Cela explique également, est-il noté, la raison pour laquelle la vague des actions des ouvriers se répercutait sur les paysans et renforçait ainsi les liens entre les populations rurale et ouvrière. Un mouvement de masse paysan avait déjà eu lieu en 1902, est-il rappelé, marqué par des insurrections dans les gouvernements de Poltava et de Kharkov. En février-mars 1905, des révoltes paysannes avaient déjà éclaté partout dans le pays. Elles s'étaient apaisées en été, le temps des travaux des champs saisonniers, pour reprendre avec force en automne. Finalement, c'étaient les révoltes dans l'armée et la marine qui avaient poussé le gouvernement à accélérer les réformes.

4. « Lenin (Ul’janov) Vladimir Il'ič », in Bol'šaja škol'naja enciklopedija « Russika »,p. 251-255.

5. A.O. Čubarjan, Otečestvennaja istorija $X X$ - načala $X X I$ veka [Histoire nationale du XXe et du début du XXI ${ }^{\mathrm{e}}$ siècle], 2004, p. 17-21. 
De façon très didactique, le manuel décrit les formes de lutte tout à fait nouvelles pour la Russie comme, par exemple, la «grève politique de toute la Russie » (vserossisjskaja političeskaja zabastovka) d'octobre 1905 à laquelle participèrent deux millions de personnes et qui paralysa les centres les plus importants de l'Empire. La même importance est accordée à l'insurrection armée de Moscou, en décembre 1905. Les exigences principales visaient, selon le manuel, à obtenir la journée de travail de huit heures, l'introduction des libertés démocratiques et la convocation d'une assemblée législative.

Le manuel accorde une grande importance aux soviets dans la mesure où non seulement ils dirigèrent les grèves, mais assumèrent aussi dans quelques villes le rôle d'organes du pouvoir. Parmi les autres structures oppositionnelles panrusses sont énumérées différentes unions professionnelles dont l'Union des paysans, fondée en mai 1905, dans laquelle les Socialistes-révolutionnaires jouèrent le rôle principal. Selon l'auteur de ce chapitre, Aleksandr Danilov, la législation et le renforcement des partis de tendances tout à fait différentes relèvent des conséquences de la révolution. À la fin de la révolution, on pouvait dénombrer environ cent cinquante partis politiques, ne représentant pourtant pas plus qu'un demi pour cent de la population. « Leur influence réelle sur la vie du pays était faible ».

Bien que l'auteur insiste sur la «formation du multipartisme russe » (formirovanie rossijskoj mnogopartijnosti), aucun des partis politiques, de droite ou de gauche, ni aucun de leurs dirigeants n'est caractérisé. À la fin du chapitre, une observation laconique rend compte de la formation de trois courants du mouvement social (obščestvennoe dviženie) au cours du «bris révolutionnaire » (revoljucionnyj razlom) : un courant traditionaliste-monarchique, un courant libéral et un courant socialiste. La lutte que ces courants se livraient entre eux « détermina le sort et l'orientation des réformes dans le pays ».

La limitation du pouvoir de l'autocratie, l'introduction des libertés politiques, la naissance des partis légaux et des unions ainsi que l'amélioration du niveau de vie des ouvriers et l'abolition des annuités de rachat des paysans sont mises au crédit de la révolution.

Dans ce chapitre, nous n'apprenons rien sur la Guerre russo-japonaise qui contribua pourtant sévèrement à ébranler l'autocratie de l'intérieur, à convaincre la société de la faiblesse du pouvoir et, par conséquent, à attiser l'atmosphère révolutionnaire dans le pays. Il n'est pas non plus question de l'intelligentsia qui s'était engagée pour la cause révolutionnaire et avait formé l'Union des unions. La demande d'une réforme de l'Église orthodoxe russe par de larges couches de la société et une partie du clergé, réforme qui aurait consisté avant tout à séparer l'Église de l'État autocratique, ne figure pas non plus parmi les problèmes sociétaux qui furent activés par la révolution.

Le fait que Lenin et les bolcheviks n'apparaissent plus comme des acteurs de la révolution est peut-être un progrès par rapport au rôle exagéré qui leur fut attribué dans les manuels soviétiques. Mais d'autres formations de gauche comme les mencheviks ou les anarchistes n'apparaissent pas non plus. Trockij qui joua un rôle important dans le soviet de Saint-Pétersbourg, à partir duquel il élabora sa théorie de 
la « révolution permanente » (l'évolution de la révolution bourgeoise à la révolution prolétarienne) n'est pas mentionné non plus, bien que son livre 1905 représente une analyse toujours valable de ces années et de la stratégie révolutionnaire ${ }^{6}$. Mais ici, comme dans d'autres manuels, la révolution n'est pas analysée d'un point de vue théorique. Ici, comme ailleurs, la révolution est représentée comme une manifestation sans révolutionnaires.

À la fin de ce chapitre consacré au «premier bris révolutionnaire » dans la sous-rubrique «Questions », le pauvre élève est invité à expliquer pourquoi les années de la première révolution russe sont considérées comme une période de la formation du multipartisme en Russie. Il lui est demandé également d'évaluer la révolution de 1905-1907 dans la perspective de l'évolution ultérieure de la Russie. Pourtant, l'étude de ce seul chapitre rend complètement impossible toute réponse.

C'est seulement dans le chapitre suivant, « Les réformes politiques. La première expérience de constitutionalisme et du parlementarisme en Russie $»^{7}$, dans lequel les événements sont d'abord décrits du point de vue du pouvoir politique, que sont évoqués aussi les partis politiques et la formation de la Douma. La «Douma de Bulygin » est évaluée comme « un premier pas sur la voie de la création d'un parlementarisme véritable» (polnocennyj) et le manifeste d'Octobre comme «un événement important (krupnoe sobytie) dans la vie politique du pays », une « percée sur la voie des réformes », un « changement important dans la configuration du pouvoir ». Une citation du journal de Nicolas II, qui prie Dieu de lui venir en aide pour sauver la Russie, des extraits du manifeste d'Octobre et des lois fondamentales de l'Empire de Russie en 1906 - tout ceci sans aucun commentaire tels sont les seuls documents historiques « originaux » qui forment la base de ce chapitre, dont l'idée principale est que, même si le pouvoir suprême exerça son contrôle sur le Parlement, le manifeste d'Octobre fut un progrès considérable dans la voie de la réforme.

La nouvelle loi électorale de la Douma y est également discutée, aussi bien que les bases du «multipartisme légal » russe. Parmi les partis, les constitutionnels démocrates, l'Union du 17 octobre, ainsi que les partis «monarchistes et traditionalistes » sont mentionnés en premier lieu tandis que les partis de gauche n'entrent pas dans ce chapitre consacré au «multipartisme ». Dans le manuel de Čubarjan, comme dans d'autres manuels, il n'est pas fait état de l'impact des programmes des partis de gauche sur la politisation des actions révolutionnaires. Si l'élève fait preuve de curiosité, il trouvera quelques brèves remarques dans le chapitre précédent (chapitre 2), «L'État et la société au début du XX $\mathrm{X}^{\mathrm{e}}$ siècle » dont

6. Lev Trockij, 1905, M. : Gos. Izd., 1922. Ce texte de 1922 était le résultat de la fusion de deux articles publiés l'un en 1906 à Saint-Pétersbourg et l'autre en 1909 à Dresde. Pour Trockij, l'insurrection d'octobre était le point culminant de la révolution de 1905, à la différence de Lenin (et plus tard aussi de Stalin) pour lesquels c'est l'insurrection de décembre qui fut décisive. Plus tard, tous ceux qui virent dans l'insurrection d'octobre l'élément culminant de 1905 furent accusés de trotskisme.

7. Čubarjan, Otečestvennaja istorija XX - načala XXI veka, p. 21-26. 
un sous-chapitre, «Le pouvoir et l'opposition politique » évoque les organisations populistes, les SR et le RSDRP. Dans le même chapitre, les organisations ouvrières légales et Gapon sont brièvement évoqués ${ }^{8}$.

Ce n'est qu'après la description des réformes stolypiniennes (chapitre 6) que, dans un chapitre intitulé «La politique extérieure de la Russie au début du $\mathrm{XX}^{\mathrm{e}}$ siècle », la Guerre russo-japonaise est évoquée, mais de manière très générale. Cette guerre est ainsi déconnectée de ses répercussions sur les événements révolutionnaires qui sont exclusivement relégués dans la « politique intérieure ». Par suite de la structuration rigide de cet ouvrage qui, comme la plupart des autres manuels, sépare strictement politique intérieure et extérieure, nous y chercherions en vain des informations sur les effets de la révolution russe dans d'autres pays. C'est seulement dans le volume présentant des documents et des sources de l'époque, qui accompagne le manuel dirigé par Čubarjan et qui a paru un an plus tard, que l'élève trouvera quelques informations sur la révolution de 1905 vue par des observateurs occidentaux.

La formulation des questions posées aux élèves à la fin de ce dernier chapitre implique que les libéraux eurent tout à fait raison de considérer le manifeste d'Octobre et les lois fondamentales comme un véritable bouleversement (perevorot) dans l'histoire de la Russie. L'autorité exercée par le tsar sur le Conseil des ministres et les ministres eux-mêmes suscite par contre une évaluation plutôt critique.

Bien que nous ayons accordé une attention particulière à ces deux représentations de 1905 - la première anodine et conventionnelle, la seconde bienveillante et libérale - il serait erroné de vouloir situer le spectre des manuels d'histoire russe entre ces deux narrations. Nous ne les avons choisis qu'à titre d'exemples qui nous serviront à observer et à comparer comment quelques-uns des problèmes évoqués (ou même ignorés) ici à partir de points de vues très différents sont traités dans d'autres manuels. Revenons donc aux questions clefs mentionnées plus haut et leur «traitement » dans les manuels.

1. Concernant la mise en perspective historique de la première révolution russe, il est exceptionnel qu'elle soit interprétée sur l'arrière-fonds du mouvement révolutionnaire russe du XIX ${ }^{\mathrm{e}}$ siècle ou qu'elle soit mise sur le même plan que les révolutions de 1917. Dans quelques rares manuels, les événements révolutionnaires de 1905 sont qualifiés de smuta («Temps des Troubles », « sédition»). La notion « révolution » n'est presque jamais explicitée ni jamais interprétée dans l'optique des révolutions européennes. À l'exception des chapitres consacrés à la politique extérieure, la Russie n'est presque jamais vue du dehors ou mise en perspective avec l'évolution des pays européens. Un point, cependant, est significatif : dans quelle période de l'histoire de la Russie range-t-on la première révolution : représente-t-elle la fin de l'époque de l'autocratie illimitée ou le début d'une nouvelle époque caractérisée par le parlementarisme et le multipartisme? 
Les manuels de civilisation russe, dont l'argumentation s'oriente en général vers la perspective de la «longue durée » des civilisations et qui présentent la Russie « en tant que tout unique » ( $v$ kačestve edinogo celogo), ce qui leur permet d'ignorer les « détails » factuels et événementiels, rangent souvent la révolution de 1905 dans la section au titre très neutre de « Fin du XIX ${ }^{\mathrm{e}}$ - début du XX $\mathrm{X}^{\mathrm{e}}$ siècle ${ }^{9}$ ou bien dans un chapitre intitulé «Problèmes de choix historique au début du XXe siècle » ${ }^{10}$. Les manuels d'histoire qui s'organisent presque exclusivement en fonction des événements politiques placent généralement la révolution de 1905 dans des chapitres intitulés «Le mouvement social à la fin du XIXe et au début du $\mathrm{XX}^{\mathrm{e}}$ siècle ${ }^{11}$, «L'instauration de la monarchie de la Douma » (Stanovlenie dumskoj monarhii) ${ }^{12}$, ou encore «L'Empire de Russie à la veille de la Première Guerre mondiale », mais on la trouve aussi traitée dans un chapitre intitulé «Épilogue d'une grande évolution : la Russie en 1900-1916 »13. Nous n'avons trouvé aucun manuel qui aurait traité la révolution de 1905 comme un chapitre principal indépendant, contrairement à ce qui avait été pratiqué dans des manuels de l'époque soviétique.

Le fait qu' «au cours du XIX siècle, de génération à génération, l'attente de la révolution comme la peur de la révolution s'était enracinée toujours plus profondément dans la conscience privée et publique »14, n'est jamais réellement thématisé. De brefs renvois au mouvement révolutionnaire se trouvent dans des chapitres précédents, et portent souvent des titres comme : « Le mouvement social et politique dans la seconde moitié des années 1890 et au début des années 1900 ». Même si l'intelligentsia révolutionnaire et ses dirigeants ou les partis révolutionnaires et leurs programmes sont signalés, c'est toujours de manière très lapidaire ${ }^{15}$. Sans doute, les manuels reflètent-ils ici une tendance générale de la Russie d'aujourd'hui, où le mouvement révolutionnaire n'intéresse plus personne et où le terme d'intelligentsia n'est réservé qu'à une élite inspirée par des valeurs spirituelles et ignorant les bassesses de la vie sociale et politique.

Ainsi il est significatif de remarquer que le mouvement révolutionnaire de l'année 1905 est évoqué indépendamment de l'octroi des lois fondamentales de l'Empire par le tsar ou de la convocation de la Douma, comme si l'un n'avait rien à

9. Voir N.V. Sisova, Istorija i kul'turologija [Histoire et culturologie], 2000.

10. Voir L.I. Semennikova, Rossija v mirovom soobščestve civilizacii [La Russie dans la communauté mondiale de la civilisation], 1994.

11. Voir A.S. Orlov, V.A. Georgiev, A.Ju. Polunov, Ju.Ja. Tereščenko, Osnovy kursa istorii [Fondements du cours d'histoire], 1999 ; A.N. Saharov, réd., Istorija Rossii, tome 2 : S načala $X I X$ veka do načala XXI veka [Histoire de la Russie du début du XIX ${ }^{\mathrm{e}}$ au début du XXI ${ }^{\mathrm{e}}$ siècle), 2005.

12. Voir V.P. Ostrovskij, A.I. Utkin, Istorija Rossii, $X X$ vek [Histoire de la Russie, $\mathrm{XX}^{\mathrm{e}}$ siècle], 2002.

13. Voir chapitre 6 in A.A. Danilov, L.G. Kosulina, Istorija Rossii. XX vek. Učebnik dlja 9ogo klass [Histoire de la Russie, XXe siècle, manuel pour la 9e classe], 1998.

14. Dietrich Geyer, Die russische Revolution, Göttingen : Vandenhoek \& Ruprecht, 1977, p. 21.

15. Par exemple, A.Ju. Dvorničenko, E.V. Il’in, Ju.V. Krivošeev, Ju.V. Tot, Russkaja istorija [Histoire de la Russie], 1999, p. 261-263. 
faire avec l'autre ${ }^{16}$. Dans nombre de manuels, la description de la révolution est restreinte à la seule année 1905, comme si l'insurrection armée à Moscou avait mis fin au tourbillon des événements et que le tout se «dissolvait harmonieusement » dans le nouveau système du multipartisme.

Il serait vain de chercher des renvois aux révolutions de 1917 ou encore des comparaisons avec des révolutions en Europe : les manuels de l'histoire nationale sont organisés de manière strictement chronologique et se réfèrent exclusivement à des événements ayant lieu en Russie même. Nous l'avons déjà souligné, la Guerre russo-japonaise n'est que rarement évoquée dans le contexte plus large de l'année révolutionnaire 1905, bien que la défaite de la flotte russe dans la bataille décisive de Tsushima qui révélait le retard de la modernisation de la Russie par rapport au Japon, se fût déroulée quasiment au même moment où avaient lieu les grands mouvements de grèves du mois de mai. Une exception cependant dans un livre préparant les élèves des classes de $9^{\mathrm{e}}$ et de $11^{\mathrm{e}}$ aux examens d'histoire où, après une description détaillée de la guerre, la question est posée de savoir dans quelle mesure cette guerre avait mobilisé le mécontentement de l'opposition à l'autocratie ${ }^{17}$.

Nous avons trouvé un seul manuel qui, avant même d'aborder 1905, demande à l'élève de réfléchir sur la différence entre « évolution » et « révolution » ainsi que sur la voie qu'il aurait lui-même préféré pour la Russie : «La révolution était-elle inévitable en Russie ? ${ }^{18}$ Selon nos enquêtes, le manuel de M.P. Irošnikov et A.Z. Vakser ${ }^{19}$, qui n'est rien d'autre qu'une sélection de sources et de documents des témoins politiques et culturels de l'époque (parmi lesquels des extraits des discours de Nicolas II, mais aussi des poèmes et souvenirs sur 1905 de K. Bal'mont, K. Paustovskij et V. Korolenko), sans commentaires, mais avec de bonnes annotations, est le seul qui envisage les trois révolutions russes (1905, Février et Octobre 1917) comme une unité. Elles sont traitées dans la partie : «Les cataclysmes politiques et militaires », où nous trouvons aussi le texte d'un appel du soviet des députés ouvriers du 17 octobre 1905 et dans les annotations un renvoi à Trockij - assez inconcevable chez les autres auteurs de manuels ${ }^{20}$.

16. Saharov, réd., Istorija Rossii, t. 2. Ici les «événements révolutionnaires de 1905 »sont traités dans le chapitre «Le mouvement social à la fin du XIX et au début du XX ${ }^{\mathrm{e}}$ siècle », les lois fondamentales de l'Empire, les partis politiques ainsi que la Première et la Deuxième Douma dans le chapitre « La Russie entre la révolution et la guerre : 1906-1914 ».

17. Ekzamennye voprosy i otvety. Istorija 9 i 11 vypusknye klassy [Questions et réponses aux examens. Histoire en $9^{\mathrm{e}}$ et $11^{\mathrm{e}}$ classes terminales], 2005, p. 388-391. Voir également une autre brochure éditée pour la préparation des examens, Primernye bilety i otvety po istorii dlja podgotovki k ustnoj attestacii vypusnikov 11 klassov obščeobrazovatel'nyh učreždenij [Questions types et réponses en histoire pour préparer l'attestation orale des écoliers], 2007, p. 18.

18. Ostrovskij, Utkin, Istorija Rossii..., p. 40 (Rubrique Dajte otvet! [Donnez votre réponse !]).

19. M.P. Irošnikov, A.Z. Vakser, Rossija $v X X$ veke: narod, vlast', voyny, revoljucii, obščestvo. Učebnaja kniga po otečestvennoj istorii [La Russie au $\mathrm{XX}^{\mathrm{e}}$ siècle : le peuple, le pouvoir, les guerres, les révolutions, la société. Manuel en histoire nationale], 2004.

20. Ibid.,p. 149-150 et p. 205, note 27. 
2. En ce qui concerne les conflits sociaux qui furent à l'origine de la révolution, le manuel édité par Zagljadin, Kozlenko, Minakov et Petrov pour la $11^{\mathrm{e}}$ classe $^{21}$ fait figure d'exception dans la mesure où la débâcle de la Guerre russo-japonaise est présentée ici comme un point de départ de la perte du prestige du régime tsariste, qui contribua ainsi à la «montée de la révolution » (rost revoljucii). Les auteurs discutent aussi en détail les conditions sociales et économiques à l'origine de la « situation révolutionnaire » : la question agraire et ouvrière, la politique de russification dans les périphéries de l'Empire, le socialisme policier, les manifestations des étudiants, les pogroms contre les juifs couverts par le pouvoir tsariste, les initiatives de l'intelligentsia libérale dans les zemstva en faveur d'une monarchie constitutionnelle. Sans nommer les dirigeants du mouvement révolutionnaire (à l'exception de Gapon), on décrit les rapports de force entre la gauche et la droite et explique les concessions du régime comme une réponse à la pression des «éléments révolutionnaires » (revoljucionnaja stihija). La leçon de ces événements que le manuel veut transmettre aux élèves est toutefois claire : «Quant aux larges couches de la population, la révolution a engendré l'illusion selon laquelle la violence puisse être une méthode efficace pour résoudre des problèmes sociaux. C'est la même illusion qui a préparé une guerre civile de très grande ampleur, en $1917-1922 »^{22}$.

Certains manuels lient avant tout le problème de la modernisation de la Russie à la question agraire. "Devenue le "clou" de la première révolution russe, la question agraire exigeait sa résolution immédiate », ce qui conduisit le gouvernement à nommer Stolypin ministre président, peut-on lire dans un manuel édité par A.A. Danilov ${ }^{23}$. Il semble que dans certains manuels les paysans occupent maintenant la place accordée auparavant aux ouvriers. Dans cette optique, il est révélateur que le premier soviet des députés des ouvriers ne soit mentionné que très rarement.

L'intelligentsia n'apparaît presque jamais porteuse d'idées révolutionnaires ${ }^{24}$. Comme on le souligne avec insistance, elle ne fait son apparition que dans le camp des libéraux qui plaident pour la monarchie constitutionnelle. Le soutien que l'intelligentsia apportait à la cause des ouvriers n'apparaît quasiment pas comme si la tendance actuelle de considérer l'intelligentsia exclusivement comme une élite spirituelle avait fait disparaître de la mémoire historique son engagement politique et social. Ainsi, la « véritable » intelligentsia est celle qui a publié en 1909 le recueil Vehi [Les jalons] qui, précisément, condamnait l'engagement révolutionnaire de l'intelligentsia en général et son activisme pendant la révolution 1905 en

21. N.V.Zagljadin, S.I. Kozlenko, S.T. Minakov, Ju.A. Petrov, Istorija otečestva $X X-$ načalo XXI veka. Učebnik dlja 11 klassa, [Histoire de la Patrie, $\mathrm{XX}^{\mathrm{e}}-$ début du $\mathrm{XXI}^{\mathrm{e}}$ siècles. Manuel la classe de 11'e $], 2003$.

22. Ibid., p. 38.

23. A.A. Danilova, éd., Rossija i mir. Učebnaja kniga po istorii, čast’ 1 [La Russie et le monde, manuel d'histoire, $1^{\text {re }}$ partie], 1994, p. 374.

24. Ainsi le «camp des révolutionnaires » incluait le prolétariat, le paysannat, les artisans, la petite bourgeoisie des villes et villages, les marchands - mais pas l'intelligentsia ; voir Ekzamennye voprosy i otvety. Istorija 9 i 11 vypusknye klassy, p. 392. 
particulier. Rares sont les manuels qui soulignent l'organisation des divers groupes professionnels de l'intelligentsia dans l'Union des unions présidée par P. Miljukov, comme les enseignants, agronomes, médecins, avocats, pharmaciens, ingénieurs, etc.

La plupart des manuels laissent de côté la question des véritables acteurs de la révolution. Le bouleversement de la société et de la sphère publique (obščestvennost') par les événements révolutionnaires, exprimé dans tant d'œuvres littéraires, artistiques et philosophiques, pour ne pas parler de la presse, n'apparaît pas davantage, de sorte que la révolution de 1905 reste presque une abstraction.

3. Les résultats ou les valeurs qui sont mis en rapport avec la révolution de 1905 sont vus avant tout dans la convocation de la Douma. La plupart des manuels évoquent les partis politiques seulement dans le contexte de l'existence de la Douma et évaluent leur existence seulement comme un résultat du manifeste d'Octobre, et non comme une manifestation des besoins concrets de la société. Un tel regard se limite évidemment aux partis libéraux et conservateurs - le parti des constitutionnels démocrates et l'Union du 17 octobre - , tandis que les partis révolutionnaires qui existaient dès avant 1905, bien que dans l'illégalité, n'entrent pas dans les chapitres consacrés à 1905. « La croissance des partis libéraux fut particulièrement stimulée par les campagnes électorales de la Douma, tandis que la croissance des partis radicaux le fut par les mouvements de masse populaires. Les partis conservateurs et défensifs ont surgi dans la période de l'apogée de la révolution quand, si l'on en croit leurs leaders, il devint nécessaire de défendre l'autocratie. $\gg^{25}$

La Douma est décrite dans les chapitres qui suivent ceux consacrés aux événements révolutionnaires et par suite, les activités de la Première et de la Deuxième Douma sont entièrement détachées du contexte de sa naissance. Il est rare que la Douma soit interpellée directement, ainsi que le fait un manuel de 1994, comme un « enfant de la révolution » (detišče revoljucii) ${ }^{26}$.

L'Église orthodoxe russe est totalement ignorée par les manuels, bien que sa séparation d'avec l'État ainsi que le changement de la politique religieuse en général eussent été vivement discutés en 1905, comme déjà au cours des années précédentes. Les problèmes confessionnels dans l'Empire ne sont pas évoqués non plus. L'hégémonie de l'Église orthodoxe qui s'étendait sur tout le territoire de l'Empire russe, c'est-à-dire aussi sur les régions des peuples non orthodoxes (constituant environ un tiers de la population ${ }^{27}$ ) et qui opprimait les vieux-croyants et les sectes, les catholiques et uniates fut en effet restreint par un oukase du tsar du 17 avril 1905, c'est-à-dire six mois avant le manifeste d'Octobre. En même

25. Zagljadin, Kozlenko, Minakov, Petrov, Istorija otečestva XX - načalo XXI veka, p. 40. Une exception : le manuel édité par Ostrovskij et Utkin, Istorija Rossii... qui traite des partis socialistes pendant la période 1905-1907 et qui publie des extraits de leurs programmes.

26. Danilova, éd., Rossija i mir...,p. 372.

27. Alexis Strycek, «La révolution de 1905 et les libertés religieuses », in François-Xavier Coquin, Céline Gervais-Francelle, éds., 1905. La première révolution russe, P. : Institut d'Études slaves, 1986, p. 45-50. 
temps, la discrimination des différentes religions était abandonnée, du moins dans les termes de la loi. Rien d'étonnant non plus si l'islam, le bouddhisme et le judaïsme ne soient pas mentionnés non plus par les manuels.

4. Les événements révolutionnaires furent-ils restreints à Moscou et à SaintPétersbourg ? Quel rôle est accordé par les manuels aux manifestations dans les périphéries de l'Empire comme en Pologne, en Finlande, dans les pays Baltes et au Caucase?

La révolte d'Ivanovo-Voznesensk, où au printemps-été 1905 des dizaines de milliers d'ouvriers du textile se mettaient en grève (ce qui conduisit à la création du premier soviet), celle d'Odessa (en liaison avec la mutinerie sur le cuirassé Potemkin à la mi-juillet 1905 qui fit environ 1260 morts), celles de Sébastopol, Kharkov, Kronstadt, mais aussi celles de Lodz, Riga, Bakou, Batoum, Tiflis, Koutaïssi sont rapidement effleurées, mais leurs répercussions sur le cours des événements ne sont pas évoquées. Les constitutionnels-démocrates sont considérés généralement de façon positive grâce à leur programme d'autodétermination nationale culturelle, qui reconnaissait les langues nationales dans le système de l'éducation, dans l'édition et l'exercice de la justice, et aussi parce qu'ils se prononçaient pour une plus grande autonomie de la Pologne et de la Finlande « dans le cadre d'un État russe indivisible». Un manuel s'approprie pourtant la critique du «camp socialiste » selon laquelle les Cadets étaient restés fidèles au concept de la grande Russie (velikoderžavnost') et ne reconnaissaient point les «droits des nations et nationalités à l'autodétermination politique ${ }^{28}$. Comme nous l'avons vu, la domination de l'Église orthodoxe dans les régions non orthodoxes de l'Empire ne rencontre aucun intérêt dans les manuels. La question juive n'est, dans les meilleurs cas, qu'effleurée.

5. La plupart des manuels d'histoire sont caractérisés par un style sévère (pour ne pas dire pédant) dû à la narration qui suit un ordre strictement chronologique et événementiel. Cette approche exclut de croiser différents facteurs et événements, dont l'interaction et la convergence sont particulièrement significatives dans le cas d'une révolution, comme nous l'avons déjà vu à propos de la Guerre russo-japonaise et les partis de gauche (que les manuels traitent dans d'autres chapitres que celui consacré à 1905). Ainsi la narration, en général plutôt lourde, se restreint à l'énumération des faits et des événements et ne permet aucune réflexion transversale. Par contre, les manuels de civilisation russe, qui sont beaucoup moins restreints par le récit épisodique et événementiel, sont aussi plus aptes à mettre la révolution de 1905 en rapport avec des phénomènes plus larges de la société russe comme le retard de la modernisation, révélé, entre autres, par la défaite de l'armée russe dans la guerre contre le Japon ${ }^{29}$, ou encore avec le système des partis

28. Comme par exemple Ostrovskij, Utkin, Istorija Rossii..., p. 61.

29. À ce propos, cf. I.I. Ionov, Rossijskaja civilizacija $I X$-načalo $X X$ veka [Civilisation russe, du IXe au début du XXe], 1998, p. 276-278. 
politiques à partir du XIX siècle. L'approche « civilisationnelle » (civilizacionnyj podhod $k$ istorii) leur permet aussi plus facilement de comparer la Russie à d'autres civilisations - en l'occurrence à celle du Japon -, en insistant toujours, bien entendu, sur la spécificité de la civilisation russe.

Les cas sont rares où les auteurs des manuels d'histoire renvoient à l'historiographie récente, à des méthodes nouvelles ou aux interprétations qui se différencient par rapport aux explications soviétiques d'autrefois, à l'exception des quelques auteurs de manuels sur la civilisation russe qui marquent leurs distances par rapport à l'historiographie soviétique, d'autant plus qu'elle n'analysait pas l'histoire en termes de civilisations ${ }^{30}$. On ne trouvera pas non plus des considérations théoriques sur la révolution (ou les révolutions) en général, pour ne pas parler d'une perspective comparative, dans les manuels destinés aux étudiants de faculté, sauf dans quelques cas exceptionnels. Dans le meilleur des cas, des problématiques sont annexées aux différents chapitres sous forme de questions posées aux élèves. Il semble pourtant souvent invraisemblable que l'élève soit capable de répondre à ces questions après avoir étudié uniquement le manuel qui les propose. Pour ne prendre qu'un exemple, quelles réponses peut-il donner aux questions de ce genre : l'explosion révolutionnaire aurait-elle pu être empêchée ? Quelles étaient les « alternatives » au développement de la situation politique en 1905 et pourquoi ces alternatives ne se sont-elles pas réalisées ? - tout ceci après avoir lu une dizaine de pages qui banalisent tant les «événements », que l'élève est incapable de toute réflexion critique ${ }^{31}$. Parfois, les livrets destinés à préparer l'élève aux examens sont plus explicites en ce qui concerne la mise au clair de la problématique des événements et de leurs répercussions politiques et idéologiques. C'est ici qu'on trouve encore des renvois au marxisme en tant qu'idéologie formatrice de l'intelligentsia russe.

La teneur documentaire et iconographique des manuels, même le papier, sont d'un aspect et d'une qualité plus que modeste. Il est rare d'y trouver, à l'exception du manuel édité en 2004 par Čubarjan, des cartes géographiques ou des matériaux statistiques récents qui soient attrayants pour l'élève d'un point de vue graphique. Quelques manuels fournissent des tableaux chronologiques, mais presque jamais de renvois bibliographiques. Souvent les manuels d'histoire du $\mathrm{XX}^{\mathrm{e}}$ siècle reproduisent à l'opposé de la page du titre le texte de l'hymne national ou des emblèmes de l'État. Les auteurs ne sont presque jamais identifiés, nous n'apprenons rien sur leur affiliation universitaire ou académique ni sur leur éventuelles publications. Pour la révolution de 1905, il faut se satisfaire en général d'une carte indiquant les zones géographiques où des révoltes et les grèves éclatèrent, ainsi que de quelques photos, souvent aussi de mauvaise qualité : Nicolas II apparaît toujours comme s'il était la figure la plus éminente de la révolution, suivi par Miljukov et parfois par Stolypin; nous n'avons trouvé qu'une photo de Lenin dans le contexte de $1905^{32}$.

30. Voir Semennikova, Rossija v mirovom soobščestve civilizacii.

31. Par exemple Ostrovskij, Utkin, Istorija Rossii..., p. 55.

32. Zagljadin, Kozlenko, Minakov, Petrov, Istorija otečestva XX - načalo XXI veka p. 43. 
Dans l'ensemble, il est difficile de s'imaginer qu'à l'époque de l'image, l'élève puisse trouver un attrait à ce genre de présentation.

6. Malgré tous ces défauts, la nouveauté des manuels de cette dernière décennie par rapport à ceux de l'époque soviétique qui soulignaient le déterminisme de l'histoire (la révolution comme nécessité du processus historique), jusqu'à ces derniers temps ${ }^{33}$, n'est que trop évidente : l'éloge de la classe ouvrière dans la révolution de 1905 a cédé le pas au parlementarisme et au multipartisme qui trouvent leur racines dans les réformes introduites à la suite de la révolution de 1905. Et plus généralement, le mythe de la révolution (et dans cette optique la révolution de 1905 comme ouverture à celle de 1917), la focalisation sur la lutte des classes en tant que moteur de l'histoire, l'importance primordiale accordée à Lenin, au «parti » bolchevique et au prolétariat comme la classe la plus progressiste - tous ses facteurs ou présupposés sont totalement absents des nouveaux manuels, bien que le mythe de la révolution ne soit pas véritablement déconstruit preuves à l'appui, mais seulement abandonné au silence. Quand Lenin est nommé, ce qui est rarement le cas, il sert d'avertissement. Mais il est significatif que les auteurs d'un manuel déjà cité préfèrent se référer à l'expérience des contemporains de la révolution plutôt qu'à leur propre jugement : «Lenin était fanatiquement dévoué à la cause de la révolution, il plaçait la fidélité à ses idées plus haut que les relations personnelles, amicales. » ${ }^{34}$ Mais les mêmes auteurs exposent plus en détail que d'autres le programme du RSDRP en soulignant la part importante de l'intelligentsia dans ce parti. Ils insistent également sur la part que prirent l'intelligentsia et les professions intellectuelles dans le parti SR dont elles étaient la force motrice. Cependant pour ces auteurs, il est aussi incontestable que les réformes politiques introduites après 1905 ne peuvent se comparer en rien aux structures parlementaires occidentales ${ }^{35}$.

Tous les manuels soulignent, même avec un certain pathos, que le manifeste d'Octobre ouvrait le chemin vers la société civile ${ }^{36}$ et l'État de droit 37 en Russie et que le multipartisme fut le résultat le plus important de la révolution de 1905. Mais ces facteurs ne sont nullement cités comme preuves que la Russie se fût trouvée sur le droit chemin vers une démocratie de type occidental - ce qu'on lit par contre dans quelques manuels à propos de la révolution de Février 1917. Si la plupart des

33. Par exemple, I.B. Berčin, I.A. Fedosov, Istorija SSSR. Učebnik dlja 9 klassa [Histoire de l'URSS, manuel pour la 9e classe], sous la réd. de M.P. Kim, 1982 ; B.A. Rybakov, Istorija SSSR. Učebnik dlja 7 klassa srednej skoly [Histoire de l'URSS, manuel pour la classe de 7e de l'école secondaire], 1985 ; V.A. Rybakov, A.M. Saharov, A.A. Preobraženskij et autres, Istorija SSSR. Učebnik dlja 7 klassa srednej školy [Histoire de l'URSS, manuel pour la classe de 7e de l'école secondaire], sous la réd. de V.A. Rybakov, 1985 (2e édition 1987) ; I.A. Fedosov, Istorija SSSR. Učebnik dlja 8 klassa srednej školy [Histoire de lURSS, manuel pour la classe de 8 e de l'école secondaire], 4 e édition, 1988.

34. Zagljadin, Kozlenko, Minakov, Petrov, Istorija otečestva XX - načalo XXI veka, p. 44.

35. Ibid., p. 51.

36. Čubarjan, réd., Otečestvennaja istorija $X X-$ načalo $X X I$ veka.

37. Saharov, réd., Istorija Rossii, t. 2, p. 369. 
auteurs soulignent l'importance du parti Cadet et voient en Miljukov une figure exemplaire ${ }^{38}$ - ce qui constitue un changement radical par rapport à l'historiographie soviétique qui rangeait les Cadets après le manifeste d'Octobre dans le camp des contre-révolutionnaires - il ne s'ensuit aucunement que le libéralisme serve de modèle pour les temps actuels. De même, les « acquis » (dostiženija) de la révolution de 1905, tels que le parlementarisme et le multipartisme, ne sont aucunement mis en rapport avec la Russie postcommuniste ni avec son chemin vers la démocratie, de sorte que l'élève citoyen n'est pas invité à s'inspirer de cette leçon de l'histoire. Comme si les auteurs des manuels, surtout les plus récents, craignaient de se référer à la révolution comme à un modèle de changement social et politique... Serait-ce la raison pour laquelle en 2005 les manifestations officielles commémorant le $100^{\mathrm{e}}$ anniversaire de la révolution de 1905 furent si rares en Russie ? Observation qui concerne également le 90e anniversaire de la révolution d'Octobre qui, en 2007, fut presque plus fortement remarquée en Occident qu'en Russie.

À l'occasion d'une conférence organisée à Paris en 1981 sur le thème «1905la première révolution russe », Marc Ferro évoquait « un vide entre le monde des chercheurs, celui de l'éducation et celui de l'école ${ }^{39}$. Son propos est malheureusement toujours valable à l'époque actuelle, où les progrès considérables de la recherche historique russe récente, aussi bien sur le plan d'accès aux sources (aux archives) que sur celui des méthodes ne se reflètent pas encore dans les manuels d'histoire ni ne touchent le traitement de la révolution de 1905. Mais il est évident qu'on ne peut pas juger de l'enseignement de l'histoire par la seule étude des manuels. En histoire comme dans d'autres disciplines, une grande partie de l'enseignement dépend de l'initiative propre de l'enseignant et en premier lieu de ses qualités pédagogiques, mais aussi de l'intégrité morale de sa personnalité, de sa volonté de transmettre des connaissances aux élèves au-delà du texte du manuel et de leur transmettre des valeurs au-delà des considérations politiques du moment.

À en juger par les conseils pédagogiques (pouročnye razrabotki) fournis par quelques auteurs de manuels aux enseignants pour les confronter à des considérations méthodologiques et parfois même des recherches et des sujets nouveaux, il existe certainement un champ de possibilités pour améliorer l'état des connaissances en histoire et son enseignement. Pourvu que la politique officielle de l'enseignement de l'histoire du Xxe siècle, qui fait appel au patriotisme d'État, comme cela se reflète dans les deux derniers manuels de A.V. Filippov s'adressant aux

38. Dans un portrait de Miljukov, on renvoie à sa thèse principale (défendu dans Očerki po istorii russkoj kul'tury) selon laquelle la Russie «indépendamment de ses particularités a traversé le siècle en suivant la voie européenne de développement », cité dans Zagljadin, Kozlenko, Minakov, op. cit., p. 40. Mais on se réfère également à un point du programme du Sojuz 17 ogo oktjabjra qui se prononce pour la conservation de «l'unité et de l'indivisibilité de l'État russe »; ibid., p. 42.

39. Marc Ferro, «La vision de 1905 dans les manuels scolaires », in Coquin, GervaisFrancelle, éds., 1905. La première révolution russe, p. 523-529. 
enseignants dans une version plus dure ${ }^{40}$ et aux élèves dans une version plus allégée ${ }^{41}$ n'aie pas de suite grave et durable.

Ce tableau de la révolution de 1905, brossé ici très succinctement, n'est, certes, qu'un aspect parmi bien d'autres qui mériteraient d'être analysés pour nous donner non seulement une idée sur la transmission des connaissances historiques en général, mais encore sur l'usage de l'histoire comme d'un outil pour la reconstruction de l'histoire nationale dont témoignent les manuels d'histoire russe depuis la dernière décennie. Dans une étude plus générale et en même temps plus systématique et analytique, Wladimir Berelowitch a finement montré les changements de l'enseignement de l'histoire après la chute du régime communiste sur l'arrière-fond de la période soviétique et de son idéologie dominante. Il a relevé, à travers l'analyse de sujets très variables, touchant à l'interprétation des périodes les plus diverses de l'histoire russe, comment dans de nombreux cas le nouveau nationalisme d'État a pris la relève de l'idéologie communiste ${ }^{42}$. Ne serait-ce que pour connaître les orientations et les sensibilités historiques et politiques de la Russie d'aujourd'hui, ses manuels d'histoire sont un baromètre précieux et révélateur, donc un sujet à suivre.

\section{CERCEC, EHESS}

Jutta.Scherrer@ehess.fr

\section{Manuels consultés, cités par ordre chronologique}

A.A. Danilov, réd., Rossija i mir. Učebnaja kniga po istorii, čast’’1, M. : Vlados, 1994 ; 6e édition revue, M. : Universitet Knižnyj dom, 2003.

L.I. Semennikova, Rossija v mirovom soobščestve civilizacii, M. : Interpraks, 1994.

A.A. Danilov, L.G. Kosulina, Istorija Rossii. XX vek. Učebnik dlja 9ogo klassa, M. : Prosveščenie 1998.

N.I. Vorožejkina, V.M. Solov’ev, M.T. Studenikin, Rasskazy po rodnoj istorii. Učebnik dlja 5ogo klassa, M. : Prosveščenie, 1998.

40. A.V. Filippov, Novejšaja istorija Rossii 1945-2006gg. Učebnik dlja učitelej [Histoire de la Russie contemporaine, 1945-2006. Manuel pour enseignants], 2007.

41. A.A. Danilov, A.I. Utkina, A.V. Filippov, réd, Istorija Rossii, 1945-2007gg. : 11 klass. Učebnik dlja učaščihsja obščeobrazovatel'nyh učreždenij [Histoire de la Russie, 1945-2007 : $11^{\mathrm{e}}$ classe. Manuel pour les élèves des écoles d'enseignement général], 2008.

42. Wladimir Berelowitch, «Les manuels d'histoire dans la Russie d'aujourd'hui : entre les vérités plurielles et le nouveau mensonge national », in J.P. Jaccard, éd., Un mensonge déconcertant ? La Russie au XXe siècle, P. : L'Harmattan, p. 203-220. Sur un autre aspect des manuels d'histoire russe - l'absence quasiment totale des notions « communisme », « socialisme » et «stalinisme » - voir Jutta Scherrer, «Der Ort des «Sozialismus-Kommunismus » in den neuen russischen Lehrbüchern der Geschichte und der Kulturologie », in Brigitte Studer, Heiko Haumann, éds., Stalinistische Subjekte. Individuum und System in der Sowjetunion und der Komintern, 1929-1953, Zürich : Chronos 2006, p. 469-482. 
A.N. Bohanov, Istorija Rossii (XIX-načalo XX veka). Učebnik dlja 8-9h klassov, M.: Russkoe slovo, 1998.

I.I. Ionov, Rossijskaja civilizacija IX-načalo XX veka. Učebnik dlja 10-11h klassov, M. : Prosveščenie, 1998.

A.Ju. Dvorničenko, E.V. Il’in, Ju.V. Krivošeev, Ju.V. Tot, Russkaja istorija s drevnejših vremen do naših dnej, 3e édition, SPb. : Lan’, 1999.

B.S. Orlov, V.A. Georgiev, A.Ju. Polunov, Ju.Ja. Tereščenko, Osnovy kursa istorii Rossii, M. : Prostor, 1999.

M.E. Glavackij, éd., Istorija Rossii XX Vek. Materialy i dokumenty dlja skol'nikov i postupajuščih v vyzy, M. : Drofa, 1999.

T.S. Georgieva, Russkaja kul'tura : istorija i sovremennost', M. : Jurajt, 1999.

N.V. Sisova, réd., Istorija i kul'turologija, 2e édition, M. : Logos, 2000.

A.A. Levandovskij, Ju.A. Cetinov, Rossija $v$ XX veke. Učebnik dlja 10-11 klassov, 5e édition, M. : Prosveščenie, 2001.

V.F. Šapovalov, Rossievedenie. Učebnoe posobie dlja vuzov, M. : Fair Press, 2001.

V.P. Ostrovskij, A.I. Utkin, Istorija Rossii. XX vek, 7e édition, M. : Drofa, 2002.

A.A. Danilov, L.G. Kosulina, Uročnye razrabotki k učebniku «Istorija Rossii. XX vek », 9 klass. Kniga dlja učitelja, M. : Prosveščenie, 2003.

N.V. Zagljadin, S.I. Kozlenko, S.T. Minakov, Ju.A. Petrov, Istorija otečestva XX - načalo XXI veka. Učebnik dlja 11 klassa, M. : Russkoe slovo, 2003.

A.S. Orlov, T.L. Šestova, Osnovy istorii Rossii. Testy, M. : Prostor, 2004.

A.O. Čubarjan, réd., Otečestvennaja istorija $X X-$ načala XXI veka. Učebnik dlja 11 ogo klassa, M. : Prosveščenie, 2004.

M.P. Irošnikov, A.Z. Vakser, Rossija $v X X$ veke: narod, vlast', vojny, revoljucii, obščestvo. Učebnaja kniga po otečestvennoj istorii, SPb. : Izdatel'stvo St-Peterburgskogo universiteta, 2004.

A.O. Čubarjan, réd., Otečestvennaja istorija XX - načala XXI veka. Dokumenty i materialy Učebnik dlja 1 logo klassa, M. : Prosveščenie, 2005.

A.N. Saharov, réd., Istorija Rossii, tome 2 : S načala XIX veka do načala XXI veka, M. : Ast, Astrel' ${ }^{\prime}$ Ermak, 2005.

R.G. Pihoja, réd., Otečestvennaja istorija. Učebnik, M. : Izdanie RAGS, 2005.

Ekzamennye voprosy i otvety. Istorija 9 i 11 vypusknye klassy, M. : Ast-Press Skola, 2005.

N.V.Zagljadin, S.T. Minakov, S.I. Kozlenko, Ju.A. Petrov, Istorija otečestva. XX vek, 5e édition, M. : Russkoe slovo, 2006.

M.Ju. Brandt, V.A. Klokov, Primernye bilety i otvety po istorii dlja podgotovki $k$ ustnoj attestacii vypusnikov 11 klassov obščeobrazovatel'nykh učrezdenij, M. : Drofa, 2007.

\section{Encyclopédie}

Bol'saja škol'naja enciklopedija « Russika ». Istorija Rossii. 20v., M. : Olma-Press, 2002. 